12

\title{
Измерение шумов интенсивности и стабильности частоты повторения импульсов Cr:ZnSe-лазера с пассивной синхронизацией мод
}

\author{
() С.О. Леонов ${ }^{1,2}$, М.П. Фролов ${ }^{1}$, Ю.В. Коростелин ${ }^{1}$, Я.К. Скасырский ${ }^{1}$, П.Ю. Федоров ${ }^{1,3}$, \\ А.С. Шелковников ${ }^{1}$, В.И. Козловский ${ }^{1}$, А.Н. Киреев ${ }^{1}$, М.А. Губин ${ }^{1}$ \\ ${ }^{1}$ Физический институт им. П.Н. Лебедева РАН, \\ 119991 Москва, Россия \\ ${ }^{2}$ МГТУ им. Н.Э. Баумана, \\ 105005 Москва, Россия \\ ${ }^{3}$ Институт горения и газовой динамики, Университет Дуйсбурга-Эссена, \\ 47057 Дуйсбург, Германия \\ e-mail: leonov.sto@gmail.com
}

Поступила в редакцию 20.12.2021 г.

В окончательной редакции 20.12.2021 г.

Принята к публикации 30.12.2021 г.

Представлены результаты измерения шумов интенсивности и стабильности частоты повторения для

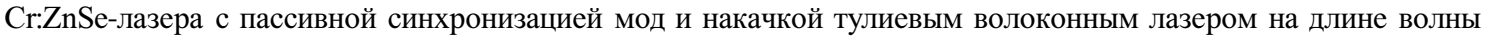
$1.94 \mu \mathrm{m}$. Проведено сравнение параметров стабильности и шумов интенсивности Cr:ZnSe-лазера для трех различных режимов генерации. Лазер работал с частотой повторения импульсов $129.5 \mathrm{MHz}$ и центральной длиной волны $2.45 \mu \mathrm{m}$.

Ключевые слова: гребенка оптических частот, синхронизация мод, шумы интенсивности, стабильность частоты повторения.

DOI: $10.21883 /$ OS.2022.04.52274.51-21

\section{Введение}

Компактные стабильные синтезаторы радиочастоты, основанные на оптическом стандарте частоты (ОСЧ), доказали свою востребованность для задач метрологии времени и частоты, навигации [1]. Кроме того, такие синтезаторы радиочастоты на основе ОСЧ могут использоваться в качестве простых лабораторных инструментов, обеспечивающих стабильную частоту в радиодиапазоне. Уже продемонстрировано несколько реализаций систем синтезирования радиочастоты на основе оптического стандарта, сущность которых заключается в использовании лазера с синхронизацией мод и сверхстабильного лазера непрерывного действия с относительной нестабильностью частоты в диапазоне от $10^{-14}$ до $10^{-18}[2,3]$. В зависимости от способа проектирования оба ключевых элемента систем синтезирования стабильной радиочастоты (стабильный непрерывный лазер и лазер с синхронизацией мод) будут обладать преимуществами и недостатками [4].

Одна из концепций создания непрерывного лазера со стабилизированной частотой основана на привязке частоты лазера к узким линиям поглощения газов [5], но в этом случае система формирует сигнал стабильной частоты в диапазоне $10^{14} \mathrm{~Hz}$. Для получения стабильной частоты в диапазоне $10^{6}-10^{9} \mathrm{~Hz}$ к стабилизированному непрерывному лазеру оптически привязывают фемтосекундный лазер, что позволяет стабилизировать частоту повторения импульсов на выходе фемтосекундного лазера $f_{\text {rep }}$ по опорной частоте непрерывного лазера [6].
Необходимо отметить, что основным фактором, ограничивающим итоговую стабильность частоты повторения, является стабильность оптического стандарта частоты, зависящая от параметров линии поглощения газа. Одним из способов повышения стабильности ОСЧ, использованного в [6], может быть переход на другие линии поглощения метана. Так, например, несколько перспективных линий метана расположены на длине волны $2.36 \mu \mathrm{m}$, а преимущество использования этих линий продемонстрировано в работе [7], где было проведено измерение ширины линии метана с помощью двухмодового непрерывного лазера на селениде цинка, легированного хромом. С другой стороны, тот же активный кристалл возможно эффективно использовать для создания лазеров с синхронизацией мод [8], которые могут быть легко оптически привязаны к стабильному непрерывному лазеру $\mathrm{Cr}: \mathrm{ZnSe}$ за счет перекрытия их спектров генерации. Для ряда применений фемтосекундный лазер используется как отдельный элемент без привязки к оптическому стандарту частоты, а основное внимание уделяется таким параметрам как шумы интенсивности и стабильность частоты повторения. На данный момент исследования таких параметров для фемтосекундных лазеров на основе твердотельного кристалла $\mathrm{Cr}: \mathrm{ZnSe}$ проведены лишь частично. В работе [9] представлены измерения шумов интенсивности для фемтосекундного лазера на кристалле $\mathrm{Cr}: \mathrm{ZnSe}$ с накачкой Er-волоконным лазером на длине волны $1.55 \mu \mathrm{m}$. Лазер работал с частотой повторения импульсов $300 \mathrm{MHz}$, длительно- 


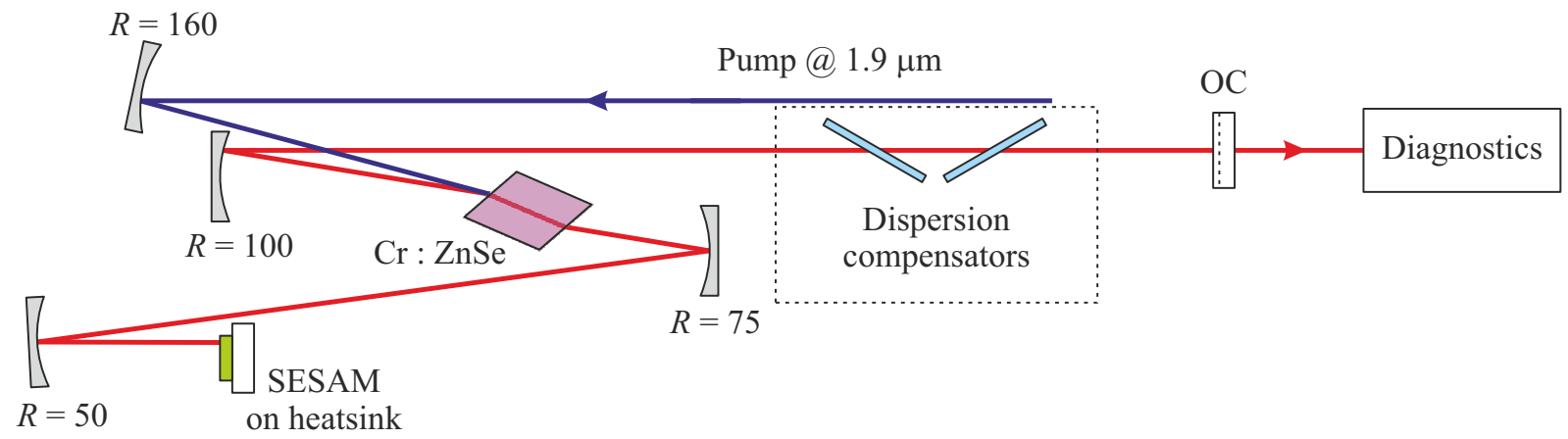

Рис. 1. Схема Cr:ZnSe-лазера с пассивной синхронизацией мод.
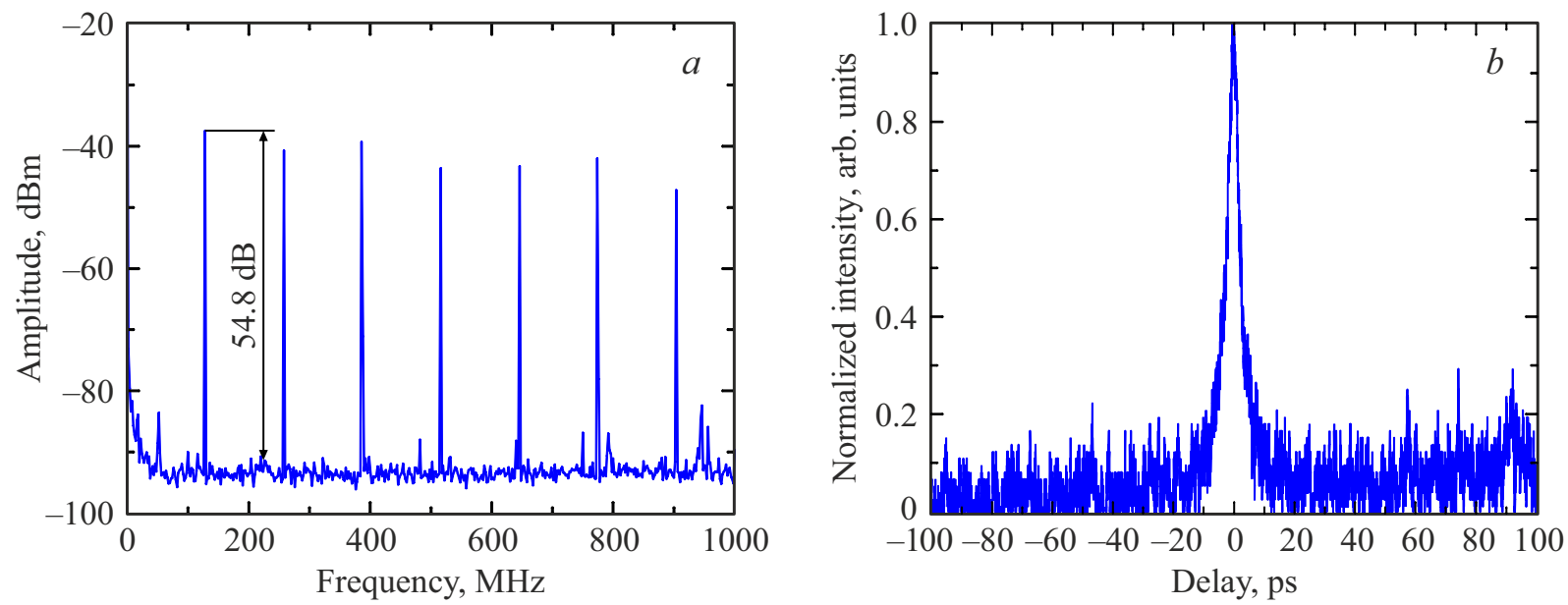

Рис. 2. Радиочастотный спектр $(a)$ и автокорреляция по интенсивности $(b)$ для режима одиночного импульса (пропускание выходного зеркала резонатора $2 \%$ ).
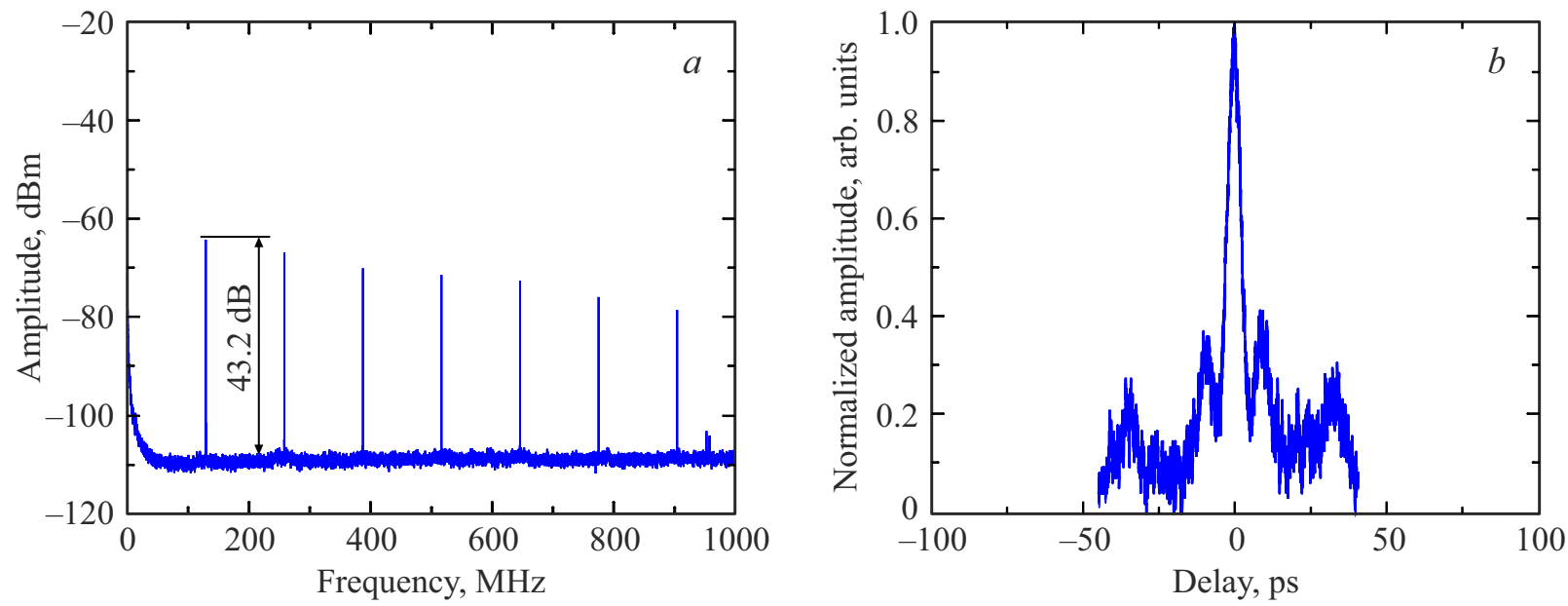

Рис. 3. Радиочастотный спектр $(a)$ и автокорреляция по интенсивности $(b)$ для режима одиночного импульса (пропускание выходного зеркала резонатора 5\%).

стью импульса $47 \mathrm{fs}$, выходной мощностью $250 \mathrm{~mW}$ и интегральным значением шума интенсивности порядка $1 \mathrm{mrad}$ в диапазоне частот от $100 \mathrm{~Hz}$ до $10 \mathrm{MHz}$, что соответствует абсолютному временному джиттеру 530 fs. Одним из недостатков данного лазера является наличие остаточных релаксационных шумов источника накачки. Авторы работы [10] использовали в качестве источника накачки лазерные диоды на длине волны $1.65 \mu \mathrm{m}$, что позволило устранить влияние релаксационных шумов волоконной накачки, хотя интегральные 

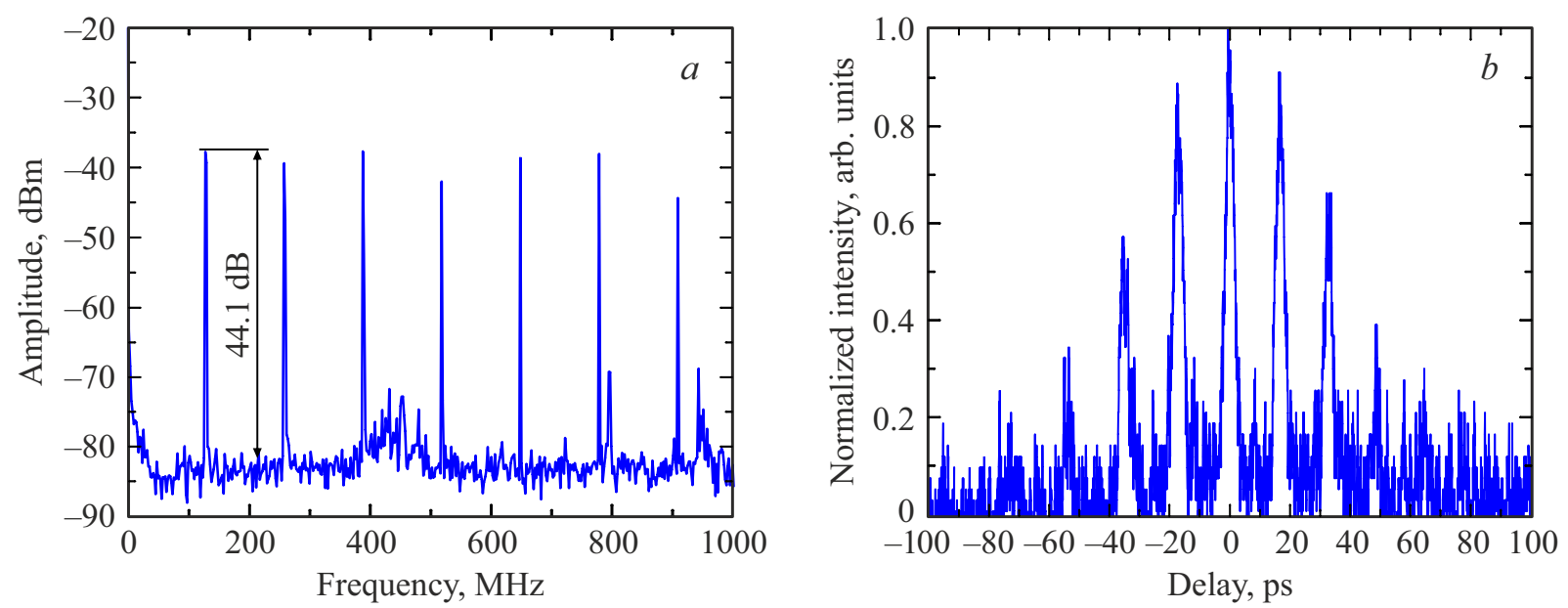

Рис. 4. Радиочастотный спектр $(a)$ и автокорреляция по интенсивности $(b)$ для режима связанных импульсов (пропускание выходного зеркала резонатора $2 \%$ ).

шумы интенсивности составили $6.8 \mathrm{mrad}$ (в диапазоне от $10 \mathrm{~Hz}$ до $1 \mathrm{MHz}$ ).

В настоящей работе проводится измерение шумов интенсивности и стабильности частоты повторения для

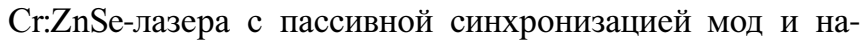
качкой тулиевым волоконным лазером на длине волны $1.94 \mu \mathrm{m}$ для трех режимов работы.

\section{Описание экспериментальной установки}

В качестве активного элемента использовался монокристалл селенида цинка, легированный ионами хрома. Кристалл был выращен из газовой фазы на подложку, с одновременным легированием ионами хрома, в Троицком обособленном подразделении Физического института им. П.Н. Лебедева РАН [11]. Схема Cr:ZnSe-лазера с пассивной синхронизацией мод представлена на рис. 1 .

Активный элемент толщиной $2.2 \mathrm{~mm}$ был закреплен в медной оправе с водяным охлаждением и размещался в Z-образном резонаторе между двумя сферическими зеркалами с радиусами 100 и $75 \mathrm{~mm}$. Для синхронизации мод использовалось полупроводниковое зеркало с насыщающимся поглотителем (SESAM). В качестве источника накачки использовался непрерывный тулиевый волоконный лазер, работающий на длине волны $1940 \mathrm{~nm}$. Для компенсации суммарной дисперсии в резонаторе использовались плоскопараллельные пластинки из фторида магния и сапфира. С пластинками из фторида магния лазер работал в режиме одиночного импульса, a c сапфировыми пластинками режим работы менялся на генерацию связанных импульсов. С подробным описанием схемы лазера и его режимов работы можно ознакомиться в работе [12].

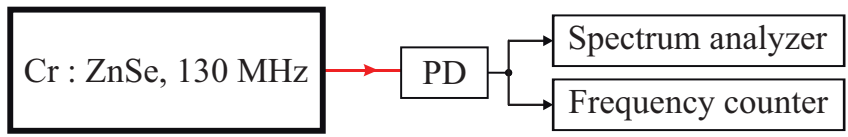

Рис. 5. Схема измерения шумов интенсивности и стабильности частоты повторения Cr:ZnSe-лазера.

\section{Результаты измерений}

Исследования шумов интенсивности и стабильности

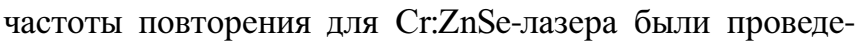
ны для нескольких конфигураций и режимов работы. Первый режим работы был реализован с компенсационными пластинками из фторида магния и выходным зеркалом лазерного резонатора с пропусканием $2 \%$. В такой конфигурации лазер работал в режиме одиночных импульсов с выходной мощностью $15 \mathrm{~mW}$, частотой повторения $129.5 \mathrm{MHz}$, отношением сигнал/шум для режима синхронизации мод $54.8 \mathrm{~dB}$ на первой гармонике радиочастотного спектра и длительностью импульса 3 ps. Центральная длина волны лазерной генерации составляла $2.453 \mu \mathrm{m}$, а ширина спектра $4 \mathrm{~nm}$. Измеренная автокорреляция интенсивности на выходе лазера и радиочастотный спектр на выходе фотоприемника приведены на рис. 2.

Второй режим был реализован с компенсационными пластинками из фторида магния и выходным зеркалом резонатора 5\%. В такой конфигурации лазер работал в режиме одиночных импульсов с выходной мощностью $45 \mathrm{~mW}$, частотой повторения $129.5 \mathrm{MHz}$, отношением сигнал/шум $43.2 \mathrm{~dB}$ и длительностью импульса $3.6 \mathrm{ps}$. Центральная длина волны лазерной генерации составляла $2.45 \mu \mathrm{m}$, а ширина спектра $3 \mathrm{~nm}$. Измеренная автокорреляция интенсивности и радиочастотный электрический спектр на выходе лазера приведены на рис. 3. 
Третий режим был реализован с компенсационными пластинками из сапфира и выходным зеркалом резонатора $2 \%$. В такой конфигурации лазер работал в режиме связанных импульсов с выходной мощностью $18 \mathrm{~mW}$, частотой повторения $129.5 \mathrm{MHz}$, отношением сигнал/шум $44.1 \mathrm{~dB}$ и длительностью одиночного импульса 2.3 ps и временным интервалом между соседними импульсами в пачке 16.7 ps. Центральная длина волны лазерной генерации составляла $2.457 \mu \mathrm{m}$, а ширина спектра $5 \mathrm{~nm}$. Измеренная автокорреляция интенсивности и радиочастотный электрический спектр на выходе лазера приведены на рис. 4.

Схема измерения шумов и стабильности частоты повторения приведена на рис. 5. Излучение лазера подавалось на быстродействующий высокочастотный приемник излучения (PD24-01-HS, IBSG Co., Ltd.), a сигнал с приемника излучения регистрировался $100 \mathrm{kHz}$-анализатором радиочастотного спектра (SR760, Stanford Research) и универсальным 350 MHzчастотомером (53230A, Keysight).

Результаты измерения шумов интенсивности для трех реализованных режимов генерации приведены на рис. 6 .

Относительная девиация Аллана была измерена на различных временах усреднения, а полученные результаты представлены на рис. 7. Кроме того, на этом же рисунке изображена кривая стабильности эрбиевого волоконного лазера, представленная в работе [13].

\section{Обсуждение результатов}

Все три режима генерации обладают достаточно высоким отношением сигнал/шум, что указывает на качество синхронизации мод, при этом наибольшее значение $54.8 \mathrm{~dB}$ реализуется для режима одиночного импульса, когда в резонаторе установлены пластинки из фторида магния и выходное зеркало с пропусканием $2 \%$. Данный режим обладает максимальным значением относительной девиации Аллана $\sigma_{y}=5.64 \cdot 10^{-8}$ для времени усреднения $1 \mathrm{~s}$, по сравнению с режимами работы, когда реализуется генерация связанных импульсов. Необходимо отметить, что изменение (с 2 до 5\%) пропускания выходного зеркала резонатора позволяет не только увеличить выходную мощность Cr:ZnSe-лазера

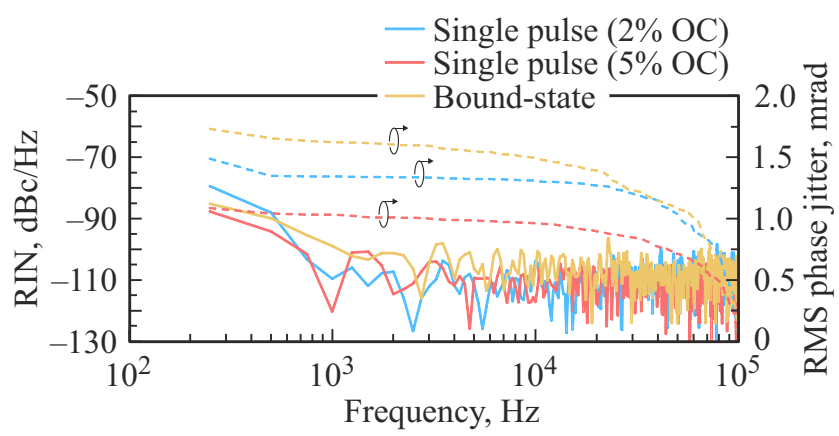

Рис. 6. Измерения шумов интенсивности для Cr:ZnSe-лазера.

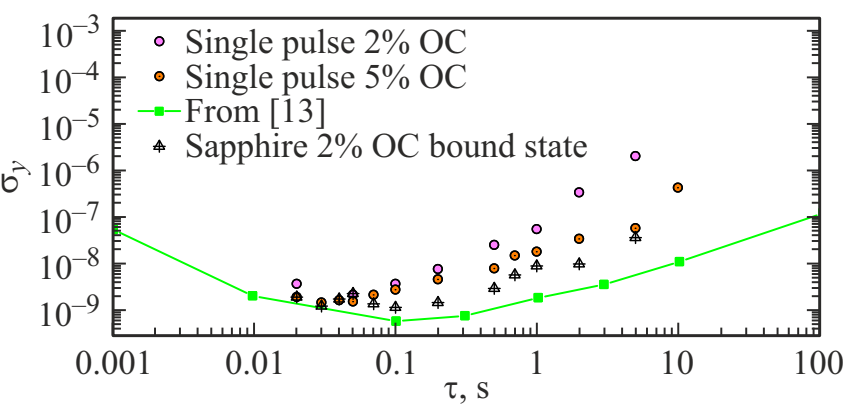

Рис. 7. Относительная девиация Аллана.

с 15 до $45 \mathrm{~mW}$ (при фиксированной мощности накачки и незначительном увеличении длительности импульса), а также улучшить стабильность частоты повторения до значения $\sigma_{y}=1.83 \cdot 10^{-8}$ для времени усреднения, равного $1 \mathrm{~s}$. Полученные результаты стабильности частоты повторения твердотельного лазера на кристалле $\mathrm{Cr}: \mathrm{ZnSe}$ показывают сопоставимость с результатами для волоконного лазера, представленными в работе [13]. Однако следует отметить, что при выполнении измерений в лаборатории не осуществлялся контроль таких параметров как температура и влажность, резонатор лазера не был изолирован специальным кожухом, а также не реализовывалась продувка азотом для уменьшения влияния поглощения на парах воды, которые содержатся в воздухе лаборатории. Таким образом, данная работа демонстрирует возможность реализации лазера на кристалле $\mathrm{Cr}: \mathrm{ZnSe}$ с пассивной синхронизацией мод в естественных условиях, что является значительным преимуществом, создающим возможность широкого применения данной технологии.

\section{Заключение}

В настоящей работе проведены измерения шумов интенсивности и стабильности частоты повторения для трех конфигураций твердотельного лазера на кристалле $\mathrm{Cr}: \mathrm{ZnSe}$ с пассивной синхронизацией мод. Наименьшее значение интегрального временного джиттера (1.5 mrad) получено для режима одиночных импульсов, когда в качестве компенсатора дисперсии используются две пластинки из фторида магния и выходного зеркала с пропусканием 2\%. Лучшая стабильность частоты повторения на уровне $9.1 \cdot 10^{-9}$ для времени усреднения $1 \mathrm{~s}$ реализуется для режима связанных импульсов, когда в качестве компенсатора дисперсии используются две сапфировых пластинки. Полученные результаты показывают, что по параметрам стабильности твердотельный и волоконный лазеры могут быть сопоставимы. Таким образом, твердотельный лазер перспективен для использования в качестве делителя оптической частоты. 


\section{Финансирование работы}

Работа выполнена за счёт средств Российского научного фонда, проект № 20-79-00155.

\section{Конфликт интересов}

Авторы заявляют, что у них нет конфликта интересов.

\section{Список литературы}

[1] F. Riehle, P. Gill, F. Arias, L. Robertsson. Metrologia, 55 (2), 188-200 (2018). DOI: 10.1088/1681-7575/aaa302

[2] X. Xie, R. Bouchand, D. Nicolodi, M. Giunta, W. Hänsel, M. Lezius, A. Joshi, S. Datta, C. Alexandre, M. Lours, P.A. Tremblin, G. Santarelli, R. Holzwarth, Y.L. Coq. Nature Photon, 11, 44-47 (2017). DOI: 10.1038/nphoton.2016.215

[3] А.С. Шелковников, А.И. Бойко, А.Н. Киреев, А.В. Таусенев, Д.А. Тюриков, Д.В. Шепелев, А.В. Конященко, М.А. Губин. Квантовая электроника, 49 (3), 272 277 (2019). DOI: 10.1070/QEL16909 [A.S. Shelkovnikov, A.I. Boiko, A.N. Kireev, A.V. Tausenev, D.A. Tyurikov, D.V. Shepelev, A.V. Konyashchenko, M.A. Gubin. Quantum Electronics, 49 (3), 272-277 (2020). DOI: $10.1070 /$ QEL16909].

[4] F. Riehle. Frequency standards: basics and applications (John Wiley \& Sons, 2006).

[5] V.L. Velichansky, M.A. Gubin. Physics-Uspekhi, 52 (11), 11531158 (2009). DOI: 10.3367/UFNe.0179.200911h.1219

[6] М.А. Губин, А.Н. Киреев, А.В. Конященко, П.Г. Крюков, А.В. Таусенев, Д.А. Тюриков, А.С. Шелковников. Квантовая электроника, 38 (7), 613-614 (2008).

DOI: 10.1070/QE2008v038n07ABEH013914 [M.A. Gubin, A.M. Kireev, A.V. Konyashchenko, P.G. Kryukov, A.V. Tausenev, D.A. Tyurikov, A.S. Shelkovnikov. Quantum Electronics, 38 (7), 613-614 (2008).

DOI: 10.1070/QE2008v038n07ABEH013914].

[7] M. Gubin et al. 2012 European Frequency and Time Forum, (IEEE 2012), pp. 459-461. DOI: 10.1109/EFTF.2012.6502425

[8] I.T. Sorokina, E. Sorokin. In: IEEE Journal of Selected Topics in Quantum Electronics, vol. 21, no. 1, pp. 273-291 (2015), Art no. 1601519. DOI: 10.1109/JSTQE.2014.2341589

[9] Y. Wang, T.T. Fernandez, N. Coluccelli, A. Gambetta, P. Laporta, G. Galzerano. Opt. Express, 25, 25193-25200 (2017). DOI: 10.1364/OE.25.025193

[10] N. Nagl, S. Gröbmeyer, V. Pervak, F. Krausz, O. Pronin, K.F. Mak. Opt. Express, 27, 24445-24454 (2019). DOI: $10.1364 / O E .27 .024445$

[11] V.A. Akimov, M.P. Frolov, Y.V. Korostelin, V.I. Kozlovsky, A.I. Landman, Y.P. Podmar'kov, A.A. Voronov. Phys. Stat. Sol. C, 3 (4), 1213-1216 (2006). DOI: 10.1002/pssc.200564723

[12] S.O. Leonov, M.P. Frolov, Y.V. Korostelin, et al. Appl. Phys. B, 127, 56 (2021). DOI: 10.1007/s00340-021-07604-X

[13] M.A. Gubin, A.N. Kireev, A.V. Tausenev, A.V. Konyashchenko, P.G. Kryukov, D.A. Tyurikov, A.S. Shelkovikov. Laser Phys., 17 (11), 1286-1291 (2007).

DOI: $10.1134 / \mathrm{S} 1054660 \mathrm{X} 07110023$ 